\title{
Self-focusing collapse distance in ultrashort pulses and measurement of nonlinear index
}

\author{
P. Whalen, ${ }^{1}$ J. V. Moloney, ${ }^{1,2}$ and M. Kolesik ${ }^{1,2,3, *}$ \\ ${ }^{1}$ College of Optical Sciences, University of Arizona, Tucson, Arizona 85721, USA \\ ${ }^{2}$ Arizona Center for Mathematical Sciences, University of Arizona, Tucson, Arizona 85721, USA \\ ${ }^{3}$ Department of Physics, Constantine the Philosopher University, Nitra 949 74, Slovakia \\ *Corresponding author: kolesik@acms.arizona.edu
}

Received May 3, 2011; accepted May 16, 2011;

posted May 18, 2011 (Doc. ID 147035); published July 1, 2011

\begin{abstract}
We analyze the method of moving focus to determine the critical power for self-focusing by means of numerical simulation and a semianalytical model. It is shown that the original interpretation of a moving focus experiment does not hold in general and that inclusion of defocusing effects due to free electrons is necessary to relate the measured data to critical power. (c) 2011 Optical Society of America

OCIS codes: $\quad 320.2250,320.5550,190.5940,190.7110,190.5530$.
\end{abstract}

The most important nonlinear mechanism underlying dynamics in high-power, ultrashort-duration optical pulses is the optical Kerr effect. It is usually quantified by the nonlinear index $n_{2}$, which relates light intensity to the change of the refractive index, $\Delta n=n_{2} I$. Alternatively, Kerr nonlinearity of a medium is often characterized by the critical power for self-focusing $\left(P_{c}\right)$. The motivation for the latter stems from the fact that if a light beam has a power higher than $P_{c}$, it will undergo a self-focusing collapse. For lower powers, diffraction overcomes selffocusing. The relation between $P_{c}$ and $n_{2}$ depends on the transverse intensity profile and has the form $P_{c} \approx \lambda^{2} / n_{2}$. Much of the ultrashort pulse dynamics depends on the peak power ratio $P / P_{c}$, and this is why it is often used to characterize both experimental and simulation setups.

Naturally, methods to determine nonlinear index values are of great practical interest. Several years ago, Liu and Chin [1] introduced a direct method to measure the critical power for self-focusing. The measurement is based on a precise determination of the propagation distance at which plasma generation reaches its maximum. This nonlinear focus position is detected from the filament luminescence captured by a camera. For peak powers below the critical power, the nonlinear focus remains unchanged and occurs close to the linear focus distance given by the lens. As the peak power exceeds the critical power, the nonlinear focus location starts to move closer to the laser. The crossover power $\left(P_{c-o}\right)$ between these two regimes is then identified as the critical power for self-focusing, $P_{c} \equiv P_{c-o}$. This method is relatively simple and direct, as it does not require calibration. Note that, in the original interpretation of this experiment, plasma generation does not play a role other than producing a source of luminescence light, which in turn reveals the focus location. In a more recent measurement of critical power in helium [2], it was observed that defocusing caused by free electrons modifies the focus also below the critical power. Nevertheless, the critical power value was still identified directly with the crossover between two distinct regimes in the focus shift.

Recently, the focus-shift method has been applied to very short, 5-6 fs duration, $800 \mathrm{~nm}$ wavelength pulses in air [3]. Laban et al. [3] reported a value for critical power as high as $18-19 \overline{\mathrm{GW}}$, which is roughly twice that measured by Liu and Chin [1] in $40 \mathrm{fs}$ duration pulses at the same wavelength. While this result might be explained by the influence of a delayed self-focusing medium response in longer pulses, the rather high value does come as a surprise and deserves attention; this provided the original motivation for the present study. To our best knowledge, the method of nonlinear focus shift was not investigated closer through simulations. In particular, it has not been verified that free-electron generation and subsequent defocusing would not affect the crossover power. If this was to happen, one could not simply identify the latter with the critical power for self-focusing, and the measurement would require a more sophisticated analysis.

Indeed, the main result of this study is that the crossover power as observed in a nonlinear focus-shift experiment cannot be directly identified with the nominal critical power for self-focusing collapse. In general, $P_{c-o}$ is significantly affected by defocusing caused by free electrons, and it also depends on the focusing geometry. Accurate determination of $P_{c}$ therefore requires help from modeling. We show that a simple semianalytic model should be sufficient. Moreover, the dependence on ionization and geometry opens a new opportunity to extract (from a set of calibrated measurements) estimates for both the nonlinear index and ionization rate.

We employ two complementary tools in this Letter. The first is computer simulation, using the unidirectional pulse propagation equation simulator [4], and the other is a semianalytic model taken from [5]. The latter represents a "minimal model," and, as such, it perhaps provides more intuitive insight than the full simulation.

The semianalytical treatment we use is based on a variational method, described in detail in [5]. It models parameters to characterize a self-focusing pulse, namely, power $P$, Gaussian beam width parameter $w(z)$, and phase front curvature. The evolution equation for the pulse width is

$$
\frac{\partial^{2} w}{\partial z^{2}}=\frac{4}{k^{2} w^{3}}\left(1-\frac{P}{P_{c}}\right)+\frac{4 K \sigma_{K} I_{0}^{K} \tau}{(K+1)^{2} k L_{\mathrm{pl}} w_{0}}\left(\frac{w_{0}}{w}\right)^{2 K+1} .
$$

This is a formula adopted for the most intense time slice of the pulse. The nonlinear index enters through the 
critical power $P_{c}$, and $k$ stands for the pulse central wavenumber. The first term on the right-hand side describes diffraction and self-focusing action due to the Kerr effect. The second term counteracts with freeelectron defocusing. Here, $K$ and $\sigma_{K}$ are an effective multiphoton ionization (MPI) order and cross-section, and $\tau$ is the effective pulse duration for the $K$ th power of intensity. $I_{0}$ and $w_{0}=w(z=0)$ stand for the initial beam intensity and width, and, finally, $L_{\mathrm{pl}}$ is the characteristic plasma length. An initial condition is completed by specifying the focal length through $w^{\prime}(0)=-w_{0} / f$.

While this model is too crude to provide an accurate picture of filamentation dynamics in general, it should capture the interplay between self-focusing, diffraction, and defocusing during the initial self-focusing collapse. This is exactly the regime we are interested in; we can calculate the position of the first minimum of $w(z)$ and identify this as the nonlinear focus location. The fact that this simple model does not work beyond the first collapse event is irrelevant for the present purpose. Indeed, comparison of results with full-blown simulations will show that the qualitative picture we obtain from this model is valid for very short pulses in which the delayed stimulated Raman effect can be neglected. The advantage in using a semianalytic model to draw our conclusion is that such analysis reduces to only those effects that play the most important role. Comparison with simulation results can be viewed as assurance that no other mechanisms affect the behavior in question.

Parameters of our numerical experiments are chosen to correspond roughly to the experiment of Laban $\mathrm{et} \mathrm{al}$. [3]. Results are shown below for the pulse duration of $6 \mathrm{fs}$ and two choices of nonlinear index $n_{2}=0.5 \times$ $10^{-23} \mathrm{~m}^{2} / \mathrm{W} n_{2}=1.0 \times 10^{-23} \mathrm{~m}^{2} / \mathrm{W}$ corresponding to critical power for self-focusing equal roughly to 18 and $9 \mathrm{GW}$, respectively. We explore a set of focal geometries characterized by a fixed focal length of $f=0.75 \mathrm{~m}$, and by initial beam waist $w_{0}$ ranging from 1.5 to $3.0 \mathrm{~mm}$. We use a parametrized power-law MPI model using values for cross-section $\sigma_{K}$ and effective ionization orders $K$ given in [6]. While both oxygen and nitrogen ionization is accounted for in our simulations, the semianalytical treatment only includes that for oxygen. Besides these reference ionization model parameters, we show results for $\sigma_{K}$ scaled by various factors in order to illustrate how behaviors depend on the relative "strength" of ionization and self-focusing. The reason we "experiment" only with the MPI power-law prefactor is that while the effective order $K$ represents the "shape" of the ionization rate as a function of intensity, and is reliably obtained from experiments, the value of $\sigma_{K}$ is less certain. We explore a very wide range (in comparison with what may be true uncertainty of measured values) of ionization rates in order to show that corresponding variations could in principle provide ways to estimate these rates.

First, we demonstrate that $P_{c-o}$ does depend on how many free electrons are generated. To this end, we artificially switch off the self-focusing effects, and we only include defocusing by free electrons. Figure 1 shows that the nonlinear focus location versus peak pulse power exhibits a very clear crossover between low- and highpower regimes. The focus shift is caused by the defocusing effect of free electrons. Higher intensity leads to

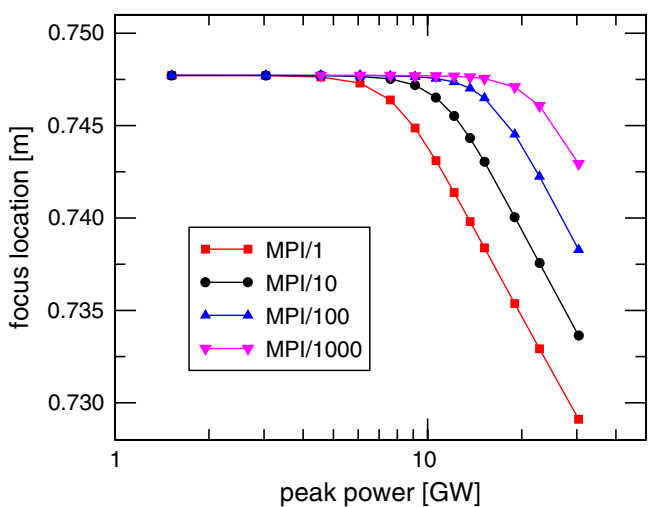

Fig. 1. (Color online) Nonlinear focus shift in ultrashort duration pulse in the absence of self-focusing effects. The clean crossover between low-power and high-power regimes is solely due to defocusing effects of the free electrons. The different curves represent results for scaled ionization rates by factors indicated in the legend.

earlier onset of ionization, and this turns a convergent beam into a divergent one before the linear focus. Because MPI is a threshold mechanism, the focus shift is tiny below a "critical" crossover power. Obviously the latter has nothing to do with the critical power for selffocusing (because the Kerr effect is switched off in these simulation runs), but it merely marks the point at which the focal intensity becomes high enough to generate a significant number of free electrons.

Now we turn to the interplay between self- and defocusing effects. Figure 2(a) shows results obtained from the semianalytic model for the reference MPI parameters and Kerr effect corresponding to the critical power $P_{c} \approx 9 \mathrm{GW}$. Different curves represent different sizes of the laser beam waist at the focusing lens. A crossover from a nearly fixed location of the focus at low power, to a high-power regime in which the focus shifts toward the laser, can be identified, as expected. However, in all cases the crossover power is significantly less than the nominal critical power. This means that the identification $P_{c} \leftrightarrow P_{c-o}$ is, in general, not valid. To show that this result is not specific to the simple model used, we show analogous results from full simulations in Fig. 2(b). The good qualitative agreement between the two approaches indicates that the simple semianalytical model contains the most important ingredients to describe the focus shift.

Comparison of the curves (in Fig. 2) obtained for various beam sizes furthermore shows that the crossover power depends on how tight the focus is. Smaller initial beams correspond to weaker focusing, which in turn means lower linear focal intensity and thus lower freeelectron production. In general, the weaker the MPI is, the closer the crossover power is to the nominal critical power. This is illustrated in Fig. 3. However, unrealistically small ionization rates would be required for $P_{c-o}$ to approach $P_{c}$.

We can see that the Kerr effect and defocusing caused by free electrons are in competition as far as the resulting location of the nonlinear focus is concerned. Both have the tendency to shift the focus toward the laser, and both contribute to the resulting focus position. The mutual interplay is strongly affected by the geometry of the experiment. Looser focal conditions weaken the effects of 


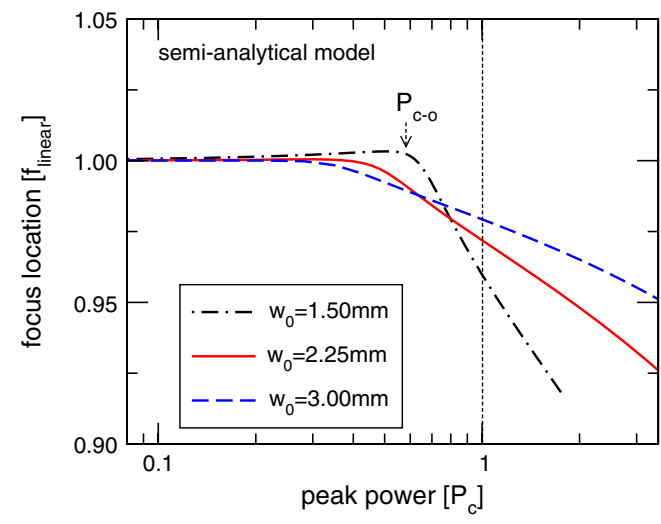

(a)

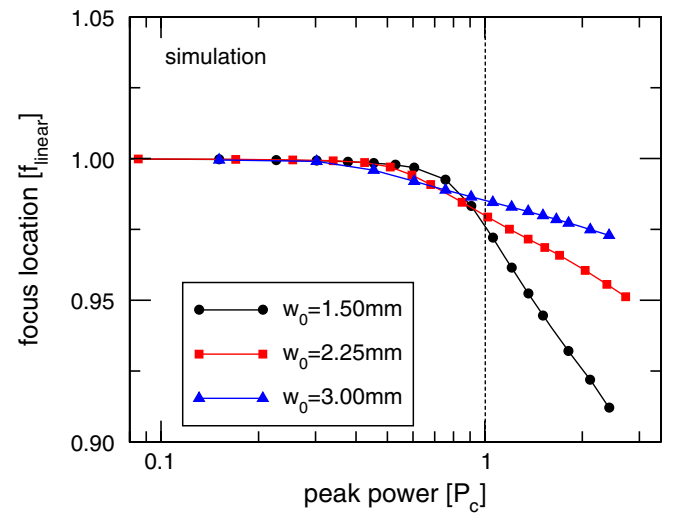

(b)

Fig. 2. (Color online) Focus position versus pulse peak power for a fixed focal length and varying beam size. In all cases, the crossover power $P_{c-o}$ appears to be significantly lower than the critical power for self-focusing $P_{c}$.

ionization, and crossover powers tend closer to the critical power. Because the detection of the focus depends on the same plasma that has the tendency to skew the results, and because tighter focusing results in more stable filamentation conditions, it is clear that optimal experimental arrangement is a matter of compromise. On one hand, one would like to minimize the effects by free electrons; on the other hand, the very detection scheme depends on them.

Thus we come to our main conclusion, namely that the moving focus method for determination of critical power for self-focusing should not be interpreted in the simple way proposed originally. Instead, plasma formation in the focal region must be taken into account not only as a means of detection, but also as an effect that contributes to the focus shift. On the positive side, this opens an

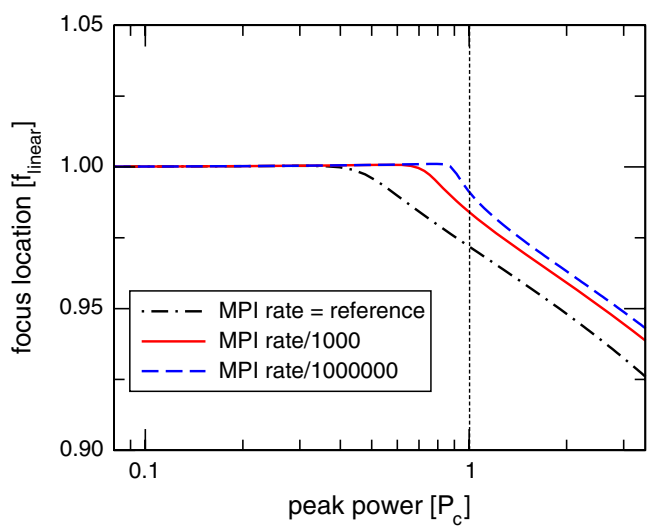

Fig. 3. (Color online) Effects of ionization rate variation: the lower the MPI rate, the higher the crossover power. Unlikely low ionization rates would be necessary for $P_{c-o}$ to approach $P_{c}$ closely.

opportunity that a set of measurements with different focal geometries together with analysis supported by numerical modeling could provide not only information about the nonlinear index, but also about the MPI rates.

An interesting issue appears in connection to the experiment by Laban et al. [3]. The very high $P_{c-o}$ measured for a few-cycle pulse seems incompatible with the ionization rates measured or calculated for longer pulses, because they would result in $P_{c-o}$ significantly lower than that measured. This may indicate that the current ionization model, or the description of effects due to free electrons may not be applicable to few-cycle pulses. Further quantitative studies with few-cycle pulses will therefore be of great value for extending our understanding of light-matter interactions on very fast time scales.

This work was supported by the Air Force Office of Scientific Research (USAFOSR) under contract FA9550-10-1-0064.

\section{References}

1. W. Liu and S. L. Chin, Opt. Express 13, 5750 (2005).

2. J. Bernhardt, P. Simard, W. Liu, H. Xu, F. Theberge, A. Azarm, J. Daigle, and S. Chin, Opt. Commun. 281, 2248 (2008).

3. D. E. Laban, W. C. Wallace, R. D. Glover, R. T. Sang, and D. Kielpinski, Opt. Lett. 35, 1653 (2010).

4. M. Kolesik and J. V. Moloney, Phys. Rev. E 70, 036604 (2004).

5. A. Couairon and A. Mysyrowicz, Phys. Rep. 441, 47 (2007).

6. J. Kasparian, R. Sauerbrey, and S. L. Chin, Appl. Phys. B 71, 877 (2000). 